\title{
A Common Language Is So Basic!
}

By: Robin Bartlett, Amanda Williams, and Rhonda Lucas

Bartlett, R., Williams, A., \& Lucas, R. (2011). A common language is so basic. Issues in Mental Health Nursing, 32 (9), 608-609.

This is an Accepted Manuscript of an article published by Taylor \& Francis in Issues in Mental Health Nursing on 23 August 2011, available online: http://www.tandfonline.com/10.3109/01612840.2011.597539

***(C) Informa Healthcare USA, Inc. Reprinted with permission. No further reproduction is authorized without written permission from Taylor \& Francis. This version of the document is not the version of record. Figures and/or pictures may be missing from this format of the document. ***

\begin{abstract}
:
It seems obvious that a person seeking care of any type should have a caregiver who speaks that person's language. Unfortunately, that does not always happen in the mono-lingual US, where a sizable minority, more than $8.5 \%$ of those aged 5 years and older, speak English "less than very well" (U.S. Census Bureau, 2009). In eight states, $10-20 \%$ of the population fall into this category, making it even more challenging for them to find a caregiver with whom they can communicate. Nursing care, especially that associated with the provision of mental health services, centers on conversation. Language barriers can compromise such care and pose a risk to patient health.
\end{abstract}

Keywords: mental health nursing | language barriers | communication | limited English proficiency (LEP) $\mid$ medical translation services

\section{Article:}

It seems obvious that a person seeking care of any type should have a caregiver who speaks that person's language. Unfortunately, that does not always happen in the mono-lingual US, where a sizable minority, more than $8.5 \%$ of those aged 5 years and older, speak English "less than very well" (U.S. Census Bureau, 2009). In eight states, $10-20 \%$ of the population fall into this category, making it even more challenging for them to find a caregiver with whom they can communicate. Nursing care, especially that associated with the provision of mental health services, centers on conversation. Language barriers can compromise such care and pose a risk to patient health.

In mental health settings, language barriers have led to higher ratings of psychopathology, misdiagnoses, and misunderstandings of symptoms, and lower levels of patient self-disclosure (Bauer \& Alegría, 2010). Beeber, Lewis, Cooper, Maxwell, and Sandelowski (2009) noted that for persons newly immigrated to the US, who often speak little or no English, receiving services that address their mental health needs may be essential for adjustment to a new land and a new 
culture. Newly-arrived immigrants to the US with limited English proficiency (LEP) are more stressed, and this can lead to poorer mental health for them (Ding \& Hargraves, 2009). Especially for a client with mental health concerns, having a caregiver who is a trained interpreter or who is teamed with a trained interpreter is important.

According to Berry-Cabán and Crespo (2008), Hispanic persons may need to develop confianza, or a trusting relationship, with their caregivers before they are comfortable discussing personal issues. Hispanic patients with LEP may more easily establish confianza when a Spanish speaking person is present during the provision of care (Jones, 2008). This is doubtless true for persons who speak other languages as well. Persons with LEP for whom Spanish is the primary language report having more positive experiences and better quality of care when they are able to communicate with a trained interpreter or Spanish-speaking nurse (Garcia, Roy, Okada, Perkins, \& Wiebe, 2004; Wu, Leventhal, Ortiz, Gonzalez, \& Forsyth, 2006; Zoucha, 1998). Spanishspeaking individuals may appreciate it when nurses with even limited Spanish skills attempt to communicate with them in their language. Their attempts may result in the person with LEP feeling more comfortable and respected. It also has been reported that Mexican-American patients feel that nurses who speak even a few Spanish words are making an effort to be personable, friendly, and attentive to their needs. Nurses are viewed as noncaring and disrespectful if they do not attempt to communicate with LEP patients and if they rush through care (Zoucha, 1998). In a review of the literature, Bauer and Alegría (2010) found some evidence that self-disclosure is facilitated in mental health settings by using trained interpreters. This, in turn, can lead to patients' satisfaction with care, which, of course, is important in mental health treatment.

It is important that the interpreter be skilled and, preferably, officially trained in interpreting (being able to convey the meaning and not just the words of the speaker). Using ad hoc persons (e.g., family members, untrained bilingual staff) as interpreters can be unwise, as has been demonstrated by several authors. This can lead to miscommunications and to potential negative consequences for the client (Bauer \& Alegría, 2010; Elderkin-Thompson, Silver, \& Waitzkin, 2001; Flores et al., 2003). In the experience of one of the authors in her work with Latinas, after she speaks to them in English and before the interpreter translates, the Latinas often quietly and affirmatively nod their heads, which she judges to be indicative of their understanding and agreement with what she said. But this is immediately followed by the translation of her remarks by her interpreter, whereupon the Latinas' affirmative nodding is replaced by serious questions concerning what she had just told them. Apparently, she had mistaken non-understanding, masked by polite smiling, for understanding. She has also found that when the Latinas have an interpreter and translated documents to read, they visibly relax.

Persons seeking mental health care cannot hope to receive effective care unless they can speak with someone who can understand their issues and who can gain their trust. While having a caregiver who attempts to speak words in the language of the client may be perceived as helpful and respectful, it is not sufficient. Except in the ideal situation where the caregiver is fluent in the client's language, an interpreter must be provided. This interpreter must be able to convey not just the words but the meanings of both the client and the caregiver. In particular, the availability of persons like bilingual staff or family members who might provide word-for-word translation and who may have limited understanding of medical terminology can lead to incorrect 
interpretation of the client's meaning and/or incorrect translations of the provider's words. Mental health service agencies must ensure that appropriately trained interpreters are available to communicate with persons with limited English proficiency. Leaders in mental health service agencies must identify the language groups in their communities and provide services to these community members in their own languages so that appropriate care can be received.

Declaration of interest: The authors report no conflicts of interest. The authors alone are responsible for the content and writing of the paper.

Edited by: Mona M. Shattell, PhD, RN

DePaul University Chicago, IL, USA

Tony MacCulloch, MEd, RN, RPN, DipCouns

AUT University, School of Nursing, Auckland, New Zealand

\section{REFERENCES}

Bauer, A. M., \& Alegría, M. (2010). Impact of patient language proficiency and interpreter service use on the quality of psychiatric care: A systematic review. Psychiatric Services, 61(8), 765-773.

Beeber, L. S., Lewis, V. S., Cooper, C., Maxwell, L., \& Sandelowski, M. (2009). Meeting the "now" need: PMH-APRN - interpreter teams provide in-home mental health intervention for depressed Latina mothers with limited English proficiency. Journal of the American Psychiatric Nurses Association, 15, 249-259.

Berry-Cabán, C. S., \& Crespo, H. (2008). Cultural competency as a skill for health care providers. Hispanic Health Care International, 6(3), 115-121.

Ding, H., \& Hargraves, L. (2009). Stress-associated poor health among adult immigrants with a language barrier in the United States. Journal of Immigrant Minority Health, 11, 446-452.

Elderkin-Thompson, V., Silver, R. C., \& Waitzkin, H. (2001). When nurses double as interpreters: A study of Spanish-speaking patients in a U.S. primary care setting. Social Science and Medicine, 52, 1343-1358.

Flores, G., Laws, M. B., Mayo, S. J., Zuckerman, B., Abreu, M., Medina, L., \& Hardt, E. J. (2003). Errors in medical interpretation and their potential clinical consequences in pediatric encounters. Pediatrics, 111(1), 6-14.

Garcia, E. A., Roy, L. C., Okada, P. J., Perkins, S. D., \& Wiebe, R. A. (2004). A comparison of the influence of hospital-trained, ad hoc, and telephone interpreters on perceived satisfaction of limited English-proficient parents presenting to a pediatric emergency department. Pediatric Emergency Care, 20(6), 373-378.

Jones, S. M. (2008). Emergency nurses' caring experiences with Mexican American patients. Journal of Emergency Nursing, 34(3), 199-204.

U.S. Census Bureau. (2009). American Community Survey: 1-Year Estimates. Retrieved from http://www.census.gov/acs/www/ 
Wu, A., Levanthal, J. M., Ortiz, J., Gonzalez, E. E., \& Forsyth, B. (2006). The interpreter as cultural educator of residents. Archives of Pediatrics Adolescent Medicine, 160, 1145-1150.

Zoucha, R. D. (1998). The experiences of Mexican Americans receiving professional nursing care: An ethnonursing study. Journal of Transcultural Nursing, 9(2), 34-44. 